\title{
Norm-Conserving Finite-Difference Beam-Propagation Method for TM Wave Analysis in Step-Index Optical Waveguides
}

Junji Yamauchi, Member, IEEE, Member, OSA, Kenji Matsubara, Takeshi Tsuda, and Hisamatsu Nakano, Fellow, IEEE

\begin{abstract}
Nonconservation of power is a perplexing problem in the propagating beam analysis of transverse magnetic (TM) waves in a $z$-variant step-index optical waveguide. To conserve the power in terms of a squared norm, a modified finite-difference (FD) formula is introduced that allows a general position of a core-cladding interface. The use of the modified formula contributes to a reduction in a field profile error caused by a staircase approximation with subsequent conservation of power, particularly for a symmetrical waveguide. To obtain the power conservation even in the analysis of an asymmetrical waveguide, a $z$-derivative of the refractive index is taken into account. An asymmetrical taper and tilted waveguides placed in parallel are investigated to validate the present technique.
\end{abstract}

Index Terms-Finite-difference (FD) methods, optical beam propagation, optical waveguides.

\section{INTRODUCTION}

$\mathbf{T}$ HE STUDY of light propagation through waveguide structures is indispensable in designing complex photonics devices. For this purpose, the beam-propagation method (BPM) has extensively been used. For the analysis of a $z$-variant structure by the semivectorial or fullvectorial BPM, one is often confronted with the problem that the power is not conserved during the propagation process. For example, in a $z$-variant slab waveguide, nonconservation of power occurs when simulating the propagating beam of transverse magnetic (TM) waves. This is due to the fact that a simple Fresnel equation, which is similar to that in transverse electric (TE) waves, is used even for the TM analysis. Vassallo [1] clearly indicated necessity of including a $z$-derivative of the refractive index along the propagation direction, i.e., $\partial_{z} n^{-2}$ term. Meanwhile, Hoekstra [2] introduced the transformation of $H$ field to $H / n$. Subsequently, Poladian and Ladouceur [3] extended Hoekstra's idea to a more general case, and succeeded in analyzing TM waves in a tilted graded-index waveguide. It should be noted, however, that the transformation technique does not work well for a step-index waveguide [4].

This paper is devoted to a detailed study on the BPM analysis of TM waves in a step-index waveguide with emphasis on power conservation. It is known that the Fresnel wave equation does not conserve the power defined by Poynting vector [5]. In this work we evaluate the power using the squared norm defined by $\int\left(1 / n^{2}\right)|H|^{2} d x$ as was used in a benchmark test [6]. In other

Manuscript received July 22, 1999; revised January 5, 2000.

The authors are with the College of Engineering, Hosei University, Tokyo 184-8584, Japan.

Publisher Item Identifier S 0733-8724(00)03741-5. words, the term "power conservation" in this paper is used in terms of the conservation of the squared norm.

Since the violation of power conservation is clearly understood in a two-dimensional waveguide, we investigate propagation characteristics in symmetrical and asymmetrical slab waveguides. We first introduce the exact propagation equation for TM waves in planar geometry, and then summarize the reason why the power is not conserved by neglecting the $z$-derivative of the refractive index. We next derive a modified finite-difference (FD) formula that allows a general position of a core-cladding interface. After demonstrating the effectiveness of the modified FD formula in the eigenmode analysis, we apply the FD formula to the propagation problem in a tilted waveguide. It is found that the use of the modified FD formula improves accuracy in terms of power conservation, particularly for a symmetrical waveguide.

To achieve power conservation even for the analysis of an asymmetrical waveguide, we solve a more exact Fresnel equation, taking into account the $\partial_{z} n^{-2}$ term. Evaluation of the $\partial_{z} n^{-2}$ term serves to conserve the power even in a strongly asymmetrical waveguide tilted from the $z$-axis. As an application of the present technique, we solve an asymmetrically tapered waveguide, which was used in a benchmark test [6]. The reciprocity of the propagation is demonstrated in terms of the guided-mode power. Furthermore, a tilted coupler composed of two parallel waveguides is investigated for several tilt angles.

Complete power-conserving analysis is made using full-wave techniques, such as the FDTD method [7], the MAFIA [8], and the finite-element method [9]. The finite-element method can also reduce a discretization error, since the grid can be defined by the geometry of the refractive index. It should be noted, however, that the full-wave techniques require extensive computational effort for the analysis of the propagating beam in an optical waveguide. In this paper, we focus our attention on the analysis of the Fresnel equation by the Crank-Nicholson scheme, since this scheme is simple to use, computationally efficient, and more importantly it has been used in many BPM algorithms in the past.

\section{Formulation AND EFFECTS OF $z$-Derivative of The REFRACTIVE INDEX}

We derive the TM-wave propagation equation for planar geometry. We start from Maxwell's equations

$$
\nabla \times \boldsymbol{E}=-j \omega \mu_{0} \boldsymbol{H}
$$




$$
\nabla \times \boldsymbol{H}=j \omega \epsilon_{0} n^{2} \boldsymbol{E}
$$

where

$\epsilon_{0}$ and $\mu_{0} \quad$ are the free space permittivity and permeability, respectively;

$\omega \quad$ is the angular frequency;

$n=\quad$ is the refractive index profile of a planar wave$n(x, z) \quad$ guide.

By defining $\boldsymbol{H}=\mathcal{H}(x, z) \hat{\boldsymbol{y}}$ and eliminating $\boldsymbol{E}$ from Maxwell's equations, we obtain the following exact wave equation for the TM wave:

$$
n^{2} \frac{\partial}{\partial z}\left(\frac{1}{n^{2}} \frac{\partial \mathcal{H}}{\partial z}\right)+n^{2} \frac{\partial}{\partial x}\left(\frac{1}{n^{2}} \frac{\partial \mathcal{H}}{\partial x}\right)+k_{0}^{2} n^{2} \mathcal{H}=0
$$

where $k_{0}=\omega / c$ is the free-space wavenumber.

We now derive a propagation equation for the TM wave under the slowly varying envelope approximation (SVEA). After expressing the field $\mathcal{H}(x, z)$ as $\mathcal{H}(x, z)=H(x, z) \exp \left(-j k_{0} n_{0} z\right)$, where $n_{0}$ is the reference refractive index to be appropriately chosen, we obtain

$$
\begin{aligned}
& n^{2} \frac{\partial}{\partial z} \frac{1}{n^{2}}\left(j k_{0} n_{0} H-\frac{\partial H}{\partial z}\right)+j k_{0} n_{0} \frac{\partial H}{\partial z} \\
& \quad=n^{2} \frac{\partial}{\partial x}\left(\frac{1}{n^{2}} \frac{\partial H}{\partial x}\right)+k_{0}^{2}\left[n^{2}(x, z)-n_{0}^{2}\right] H .
\end{aligned}
$$

Since the relation $\left|j k_{0} n_{0} H\right| \gg|\partial H / \partial z|$ is maintained within the framework of the SVEA, (4) can be reduced to the following Fresnel equation:

$$
\sigma \frac{\partial H}{\partial z}=n^{2} \frac{\partial}{\partial x}\left(\frac{1}{n^{2}} \frac{\partial H}{\partial x}\right)+\left\{\nu-\frac{\sigma}{2} n^{2} \frac{\partial}{\partial z}\left(\frac{1}{n^{2}}\right)\right\} H
$$

where $\sigma=2 j k_{0} n_{0}$ and $\nu=k_{0}^{2}\left[n^{2}(x, z)-n_{0}^{2}\right]$. Equation (5) is the basis of the present work and is solved by the Crank-Nicholson scheme. The finite-difference equation becomes

$$
\begin{gathered}
{\left[\sigma-\frac{\Delta z}{2}\left\{\delta_{x}^{2}+\nu-\frac{\sigma}{2} n^{2} \frac{\partial}{\partial z}\left(\frac{1}{n^{2}}\right)\right\}\right]^{l+1} H^{l+1}} \\
=\left[\sigma+\frac{\Delta z}{2}\left\{\delta_{x}^{2}+\nu-\frac{\sigma}{2} n^{2} \frac{\partial}{\partial z}\left(\frac{1}{n^{2}}\right)\right\}\right]^{l} H^{l}
\end{gathered}
$$

where $\delta_{x}^{2}$ is the finite difference for the second derivative, which will be introduced in Section III.

The $\partial_{z} n^{-2}$ term in (5) has often been neglected in previous works. Vassallo [1] has indicated that neglecting the $z$-derivative of the refractive index causes two problems: a field profile error and nonconservation of power. Fortunately, the field profile error is not significant within the framework of the paraxial approximation. It should be noted, however, that the conservation of power is seriously violated. We, therefore, focus our attention on power conservation.

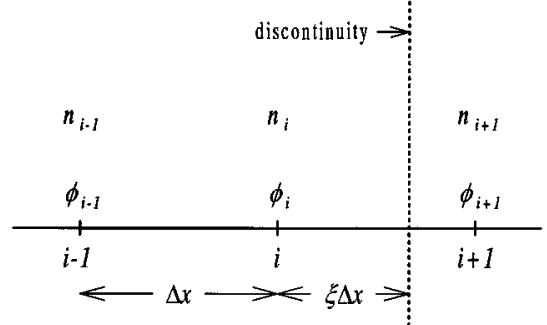

Fig. 1. Sampling points near a discontinuity.

Vassallo [1] has derived the properties of $\beta$ when the TM wave propagates in a step-index slab waveguide tilted by an angle $\Theta$ from the $z$-axis, neglecting the $z$-derivative of the refractive index in (5)

$$
2 \tilde{\beta} \int \frac{|\phi|^{2}}{n^{2}} d X=-\tan \Theta \sum_{\text {discont. }}|\phi|^{2}\left|\frac{1}{n^{2}}\right|_{-}^{+}
$$

where $\phi(X)$ and $\beta$ satisfy $\phi^{\prime \prime}(X)+\left(k_{0}^{2} n^{2}(X)-\beta^{2}\right) \phi(X)=0$, with the continuity of $n^{-2}\left(\phi^{\prime}-j \beta \phi \tan \Theta\right)$ at core-cladding interfaces, and $\tilde{\beta}$ is the imaginary part of $\beta$. The sum is performed over the discontinuities of $n(X)$ and the $\left|n^{-2}\right|_{-}^{+}$terms are the jumps of $n^{-2}$. Note that the coordinate $X$ is taken to be normal to the core-cladding interface, i.e., $X=x \cos \Theta-z \sin \Theta$.

Needless to say, the appearance of $\tilde{\beta}$ results in loss or amplification of the approximated fields. This is particularly obvious when the refractive index profile is asymmetrical. At first sight, the power seems to be conserved for a symmetrical waveguide, since the term $\sum|\phi|^{2}\left|\left(1 / n^{2}\right)\right|_{-}^{+}$is cancelled. Actual calculation, however, shows that the field is lossy or amplified as soon as the field $\phi$ contains an error (with its symmetry being lost) due to a staircase approximation. In other words, the power for a symmetrical waveguide must be well conserved, provided that the field is evaluated more properly without using the staircase approximation. This fact will be verified in Section III.

\section{Modified Finite-Difference FormUla FOR A GenERAL POSITION OF AN INTERFACE}

It can be said from the discussion in Section II that reducing the discretization error is crucial to power conservation. We should recall that the discretization error has successfully been reduced in the eigenmode analysis by the aid of a modified FD formula for a general position of an interface [4], [10]. Since the field profile error caused by the negligence of $\partial_{z} n^{-2}$ is small [1], we are encouraged to employ the modified FD formula even in the propagating beam analysis of $z$-variant structures. In particular, for a symmetrical waveguide, the power must be conserved even if we neglect $\partial_{z} n^{-2}$, as long as the discretization error is substantially reduced. In this section, we describe how the modified FD formula contributes to a reduction in the discretization error with subsequent power conservation for a symmetrical waveguide.

Three consecutive sampling points shown in Fig. 1 are considered, in which the interface of different indexes is located at distance $\xi \Delta x$ (with $0<\xi<1$ ) from point $i$. We assume that the interface is perpendicular to the $x$-axis. This assumption is approximate when treating a tilted waveguide. Nevertheless, we 


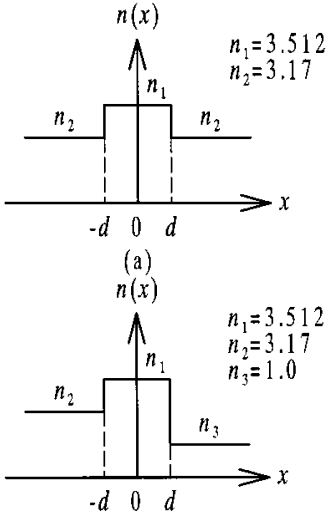

(b)

Fig. 2. Configuration of (a) symmetrical and (b) asymmetrical slab waveguides.

can expect improvement in accuracy within the framework of the paraxial approximation, as will be seen later.

Starting from the one-dimensional (1-D) Helmholtz equation, we first express the fields $H_{i-1}$ and $H_{i+1}$ based on Taylor-series expansions, as described in [4], [10], and [11]. Using the continuity relations at the interface, we obtain [10]

$$
\begin{aligned}
\left.\frac{\partial^{2} H}{\partial x^{2}}\right|_{i}= & \frac{a_{2} H_{i-1}+b_{2} H_{i}+c_{2} H_{i+1}}{d_{2} \Delta x^{2}}+\left.e_{2} \frac{\Delta x}{3} \frac{\partial^{3} H}{\partial x^{3}}\right|_{i} \\
& +O\left(\Delta x^{2}\right)
\end{aligned}
$$

where

$$
\begin{aligned}
& a_{2}=1+(\theta-1)(1-\xi)+m \Delta x^{2} \Gamma \\
& b_{2}=-2-(\theta-1)(1-\xi)-m \Delta x^{2}(1+\Gamma) \\
& c_{2}=1 \\
& d_{2}=1+\frac{1}{2}(\theta-1)(1-\xi)(2 \xi+1)+\frac{1}{2} m \Delta x^{2} \Gamma \\
& e_{2}=(\theta-1) \xi(\xi-1)(2 \xi-1) / d_{2}
\end{aligned}
$$

in which $\theta=n_{i+1}^{2} / n_{i}^{2}, m=k_{0}^{2}(1-\xi)^{2}\left(n_{i}^{2}-n_{i+1}^{2}\right) / 2$, and $\Gamma=\xi+\theta(1-\xi) / 3$.

Incidentally, the formula for the first derivative, which will be required to calculate the adaptive reference index in (12), is given by

$$
\left.\frac{\partial H}{\partial x}\right|_{i}=\frac{a_{1} H_{i-1}+b_{1} H_{i}+c_{1} H_{i+1}}{d_{1} \Delta x}+O\left(\Delta x^{2}\right)
$$

where

$$
\begin{aligned}
& a_{1}=-1+2 \xi(1-\theta)(1-\xi) \\
& b_{1}=2 \xi(\theta-1)(1-\xi)-m \Delta x^{2} \\
& c_{1}=1 \\
& d_{1}=2+(\theta-1)(1-\xi)(2 \xi+1)+m \Delta x^{2} \Gamma .
\end{aligned}
$$

Similarly, we can also derive modified FD formulas when the discontinuity lies between points $i-1$ and $i$. In this case, the coefficients are changed to

$$
\begin{aligned}
& a_{2}=1 \\
& b_{2}=-2-\left(\theta^{\prime}-1\right) \xi-m^{\prime} \Delta x^{2}\left(1+\Gamma^{\prime}\right) \\
& c_{2}=1+\left(\theta^{\prime}-1\right) \xi+m^{\prime} \Delta x^{2} \Gamma^{\prime}
\end{aligned}
$$

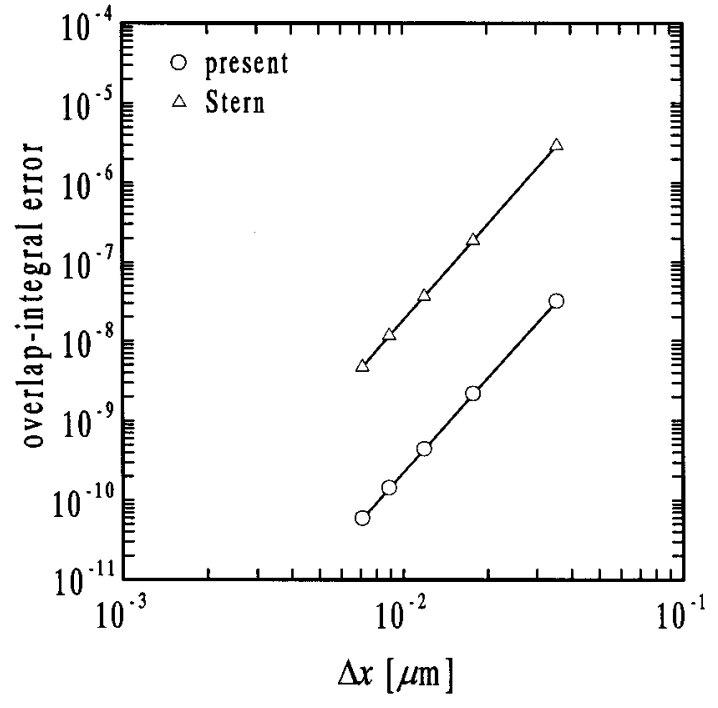

(a)

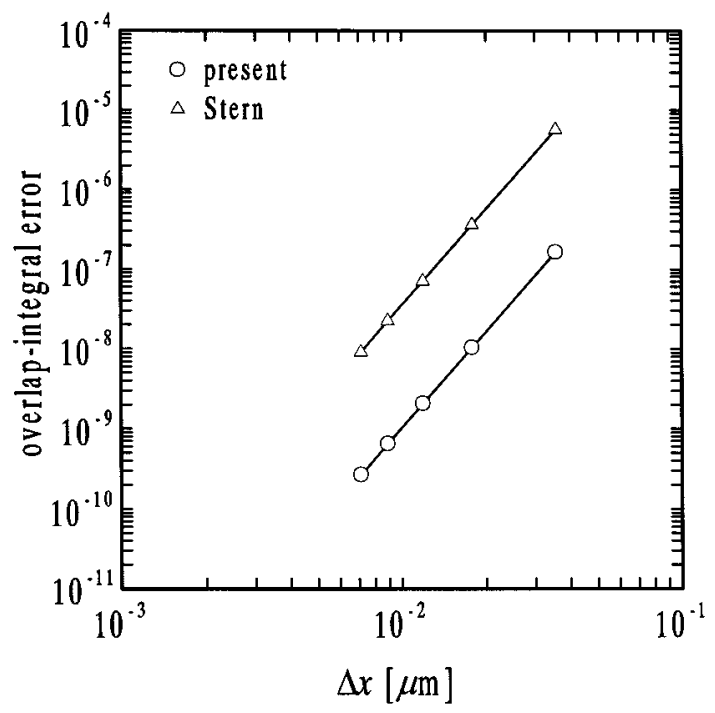

(b)

Fig. 3. Overlap integral error as a function of transverse sampling width $\Delta x$ : (a) symmetrical slab and (b) asymmetrical slab.

$$
\begin{aligned}
& d_{2}=1+\frac{1}{2}\left(\theta^{\prime}-1\right) \xi(3-2 \xi)+\frac{1}{2} m^{\prime} \Delta x^{2} \Gamma^{\prime} \\
& e_{2}=\left(\theta^{\prime}-1\right) \xi(\xi-1)(2 \xi-1) / d_{2} \\
& a_{1}=-1 \\
& b_{1}=-2 \xi\left(\theta^{\prime}-1\right)(1-\xi)-m^{\prime} \Delta x^{2} \\
& c_{1}=1-2 \xi\left(1-\theta^{\prime}\right)(1-\xi) \\
& d_{1}=2+\left(\theta^{\prime}-1\right) \xi(3-2 \xi)+m^{\prime} \Delta x^{2} \Gamma^{\prime}
\end{aligned}
$$

in which $\theta^{\prime}=n_{i-1}^{2} / n_{i}^{2}, m^{\prime}=k_{0}^{2} \xi^{2}\left(n_{i}^{2}-n_{i-1}^{2}\right) / 2$, and $\Gamma^{\prime}=$ $(1-\xi)+\theta^{\prime} \xi / 3$.

One may notice that the present FD schemes are asymmetrical near the core-cladding interface, resulting in slight loss or gain even for a straight waveguide. Preliminary calculation, however, shows that the spurious loss or gain caused by the asymmetry is much smaller than that caused by the staircase approximation or the negligence of $\partial_{z} n^{-2}$ term in a $z$-variant waveguide. For example, the spurious gain is about $5 \times 10^{-8} \mathrm{~dB}$ 


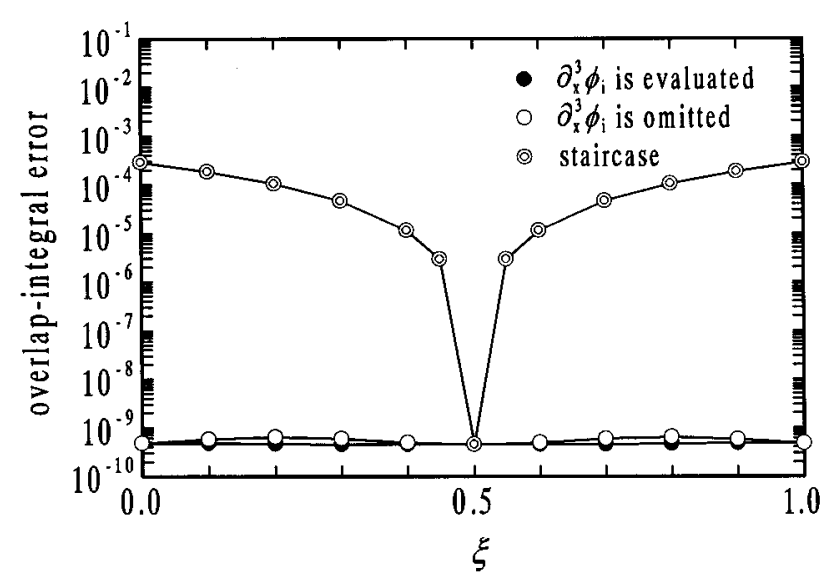

(a)

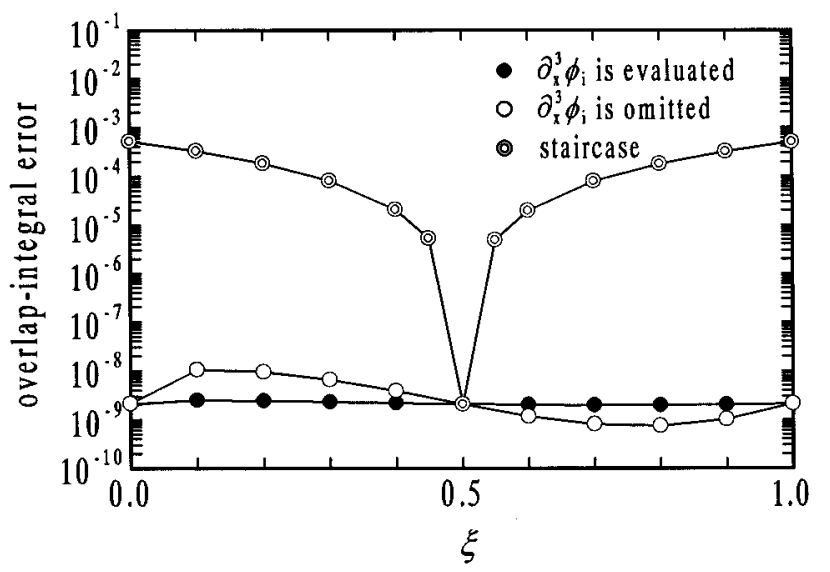

(b)

Fig. 4. Overlap integral error as a function of interface position $\xi$ : (a) symmetrical slab and (b) asymmetrical slab.

at a propagation distance of $50 \mu \mathrm{m}$ for the symmetric waveguide in Fig. 2(a), which will be found to be negligible in Fig. 6(b).

The third derivative in (8) can be evaluated using either the Helmholtz or Fresnel equation [12]. With the Helmholtz equation, the second term in the right-hand side (RHS) in (8) becomes $\left[\left(k_{0}^{2} n_{i-1}^{2}-\beta^{2}\right) H_{i-1}-\left(k_{0}^{2} n_{i}^{2}-\beta^{2}\right) H_{i}\right] e_{2} / 3$ for a discontinuity between $i$ and $i+1$ or $\left[\left(k_{0}^{2} n_{i}^{2}-\beta^{2}\right) H_{i}-\left(k_{0}^{2} n_{i+1}^{2}-\right.\right.$ $\left.\left.\beta^{2}\right) H_{i+1}\right] e_{2} / 3$ for a discontinuity between $i-1$ and $i$. The alternative technique based on the Fresnel equation is suitable if no information on $\beta$ is available [12]. Although all the data in the following are generated by the technique based on the Helmholtz equation, the effects of the third-derivative term are much smaller than those caused by the discretization error in the staircase approximation. It follows that this term may be neglected, as will be shown in Figs. 4 and 5.

We now demonstrate the effectiveness of the modified FD formula, comparing it with the well-known Stern formula [13]. Before discussing propagating beam problems, we assess the accuracy of each formula through the eigenmode analysis. Both symmetrical and asymmetrical waveguides shown in Fig. 2 are treated. The refractive indexes are $n_{1}=3.512, n_{2}=3.17$, and $n_{3}=1.0$, respectively. The core width is taken to be $2 d=$ $0.5 \mu \mathrm{m}$, and the fundamental mode at a wavelength of $\lambda=$

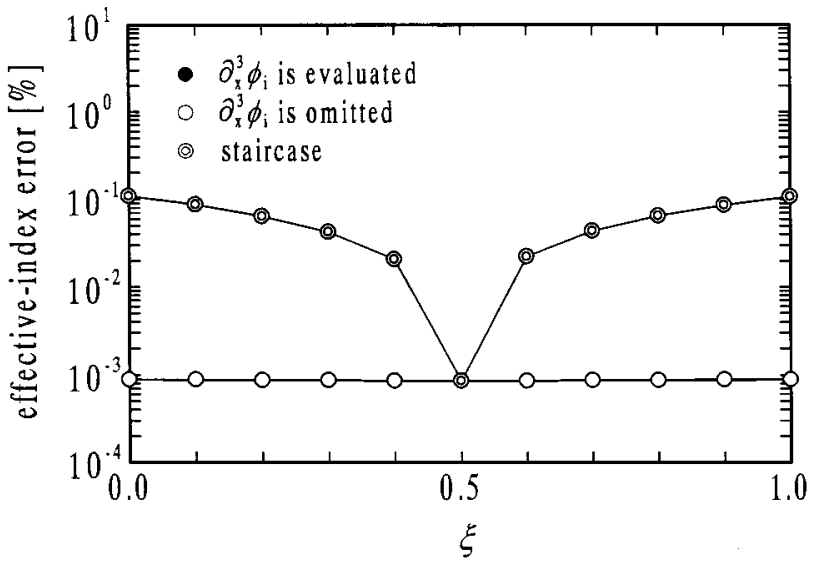

(a)

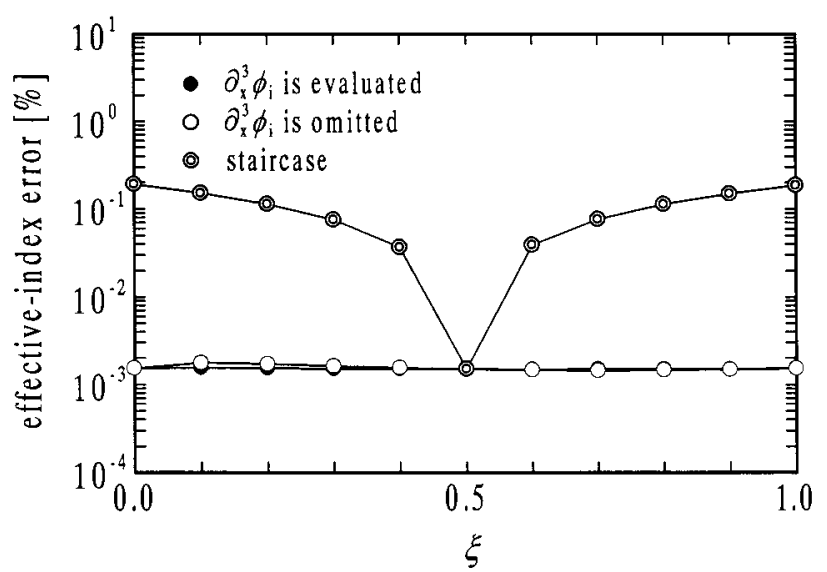

(b)

Fig. 5. Effective-index error as a function of interface position $\xi$ : (a) symmetrical slab and (b) asymmetrical slab.

$1.55 \mu \mathrm{m}$ is launched. The modal profile is determined by the imaginary distance procedure [14]-[16].

Fig. 3 shows the numerical error evaluated by the overlap integral between the numerical and exact fields as a function of transverse sampling width $\Delta x$. For comparison, the data obtained by the Stern formula are also plotted. In this analysis, $\xi$ is fixed to be 0.5 , since the Stern formula is valid only for $\xi=0.5$. It is found that the present modified formula is more accurate than the Stern one.

The advantage of the modified formula lies in the fact that the interface position can arbitrarily be chosen without moving a sampling grid. Although this advantage will be clearly found in Fig. 6, we first demonstrate the effectiveness of the present formula, while checking the effects caused by a staircase error in the core width. Investigation is made in terms of the overlap integral error and the effective index error. For the overlap integral, an appreciable error occurs when a reference waveguide is connected to the same size waveguide with a lateral offset by the order of $\Delta x$. For the effective index, an appreciable error occurs when a reference waveguide is connected to a waveguide whose core width differs from that of the reference waveguide by the order of $\Delta x$.

Fig. 4 shows the overlap integral error as a function of interface position $\xi$. In this analysis, $\Delta x$ is fixed to be $d / 21$. For 


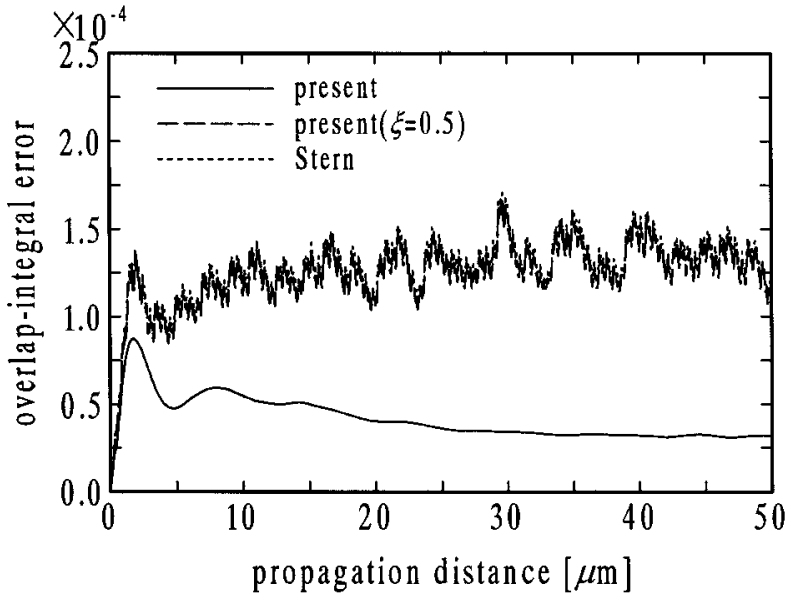

(a)

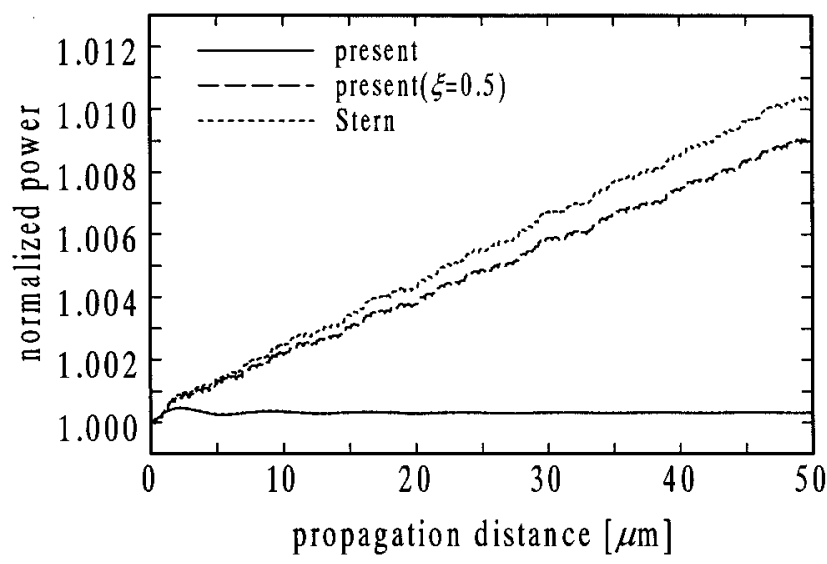

(b)

Fig. 6. (a) Overlap integral error and (b) normalized power as a function of propagation distance (symmetrical slab).

comparison, the data obtained by the staircase approximation are also plotted, in which $\xi=0$ corresponds to the data when the field in the connected waveguide is shifted toward the $+x$ direction by $0.5 \Delta x$, while $\xi=1$ when shifted toward the $-x$ direction by $0.5 \Delta x$. Although evaluation of the third derivative almost eliminates the dependency of the numerical results on the interface position, the effect is much smaller than the error caused by the staircase approximation even in the asymmetrical waveguide.

Fig. 5 shows the effective index error as a function of interface position $\xi$. The data for the staircase approximation are again plotted, in which $\xi=0$ corresponds to the data when the core width of the connected waveguide is smaller than that of the reference waveguide by $\Delta x$, while $\xi=1$ when the core width is larger than that of the reference waveguide by $\Delta x$. The behavior of numerical results is similar to those in Fig. 4.

We now turn to the propagating beam analysis. From the discussion made in the last part of Section II, we can expect that the power is conserved for a symmetrical waveguide tilted by an angle $\Theta$, provided that the discretization error is substantially reduced. To verify this fact, we calculate the normalized power together with the overlap integral error as a function of propagation distance. Fig. 6(a) and (b) shows these data for $\Theta=5^{\circ}$.

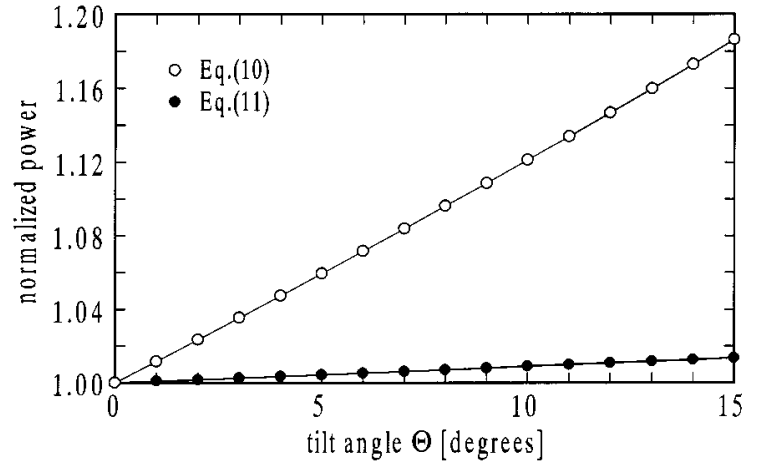

Fig. 7. Normalized power as a function of tilt angle $\Theta$ (asymmetrical slab).

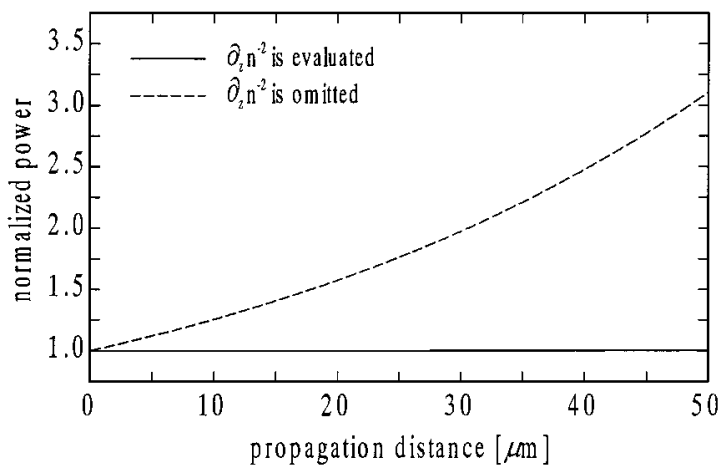

Fig. 8. Normalized power as a function of propagation distance (asymmetrical slab).

For reference, the data for $\xi=0.5$ corresponding to the staircase approximation and for the Stern formula are also presented. The sampling widths are taken to be $\Delta x=d /(21 \cos \Theta)$ and $\Delta z=0.05 \mu \mathrm{m}$. The reference index is chosen to be $n_{0}=n_{2}$. At the edge of the computational window, Hadley's transparent boundary condition is imposed [17]. Fig. 6 indicates that improvement of the present formula over the Stern one is slight as long as the staircase approximation $(\xi=0.5)$ is adopted. In other words, the formulas based on the staircase approximation make a fatal error. It is worth mentioning that the modified formula for a general position of an interface greatly contributes not only to a reduction in the overlap-integral error but also to conservation of power.

One may have some interest in comparing the present scheme with that derived by Poladian and Ladouceur [3]. Note that the scheme in [3] only holds true for a graded-index waveguide. If we blindly apply the scheme in [3] to the step-index waveguide in this section, the overlap-integral error and the normalized power are, respectively, in the order of $10^{-3}$ and 0.9 at a propagation distance of $50 \mu \mathrm{m}$, which are out of scale in Fig. 6.

\section{EVAluATION OF $z$-DERIVATIVE OF THE REFRACTIVE INDEX}

It is clear that the evaluation of the $z$-derivative of the refractive index in the TM-wave Fresnel equation is absolutely necessary to conserve the power for an asymmetrical waveguide. In this section, we try to evaluate $\partial_{z} n^{-2}$, although only an approximate evaluation is available because of discontinuous profile of a step-index waveguide. 


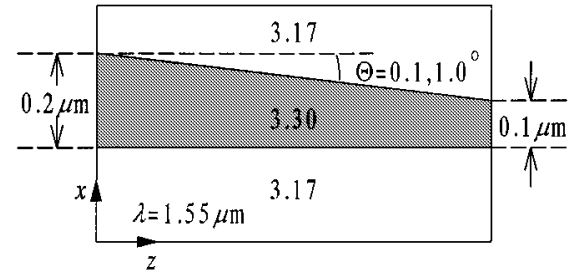

Fig. 9. Geometry of asymmetrically tapered waveguide.

One way is to evaluate it directly using the finite difference formula. Application of the central difference formula to the $z$-derivative of the refractive index leads to

$$
n^{2} \frac{\partial}{\partial z}\left(\frac{1}{n^{2}}\right)_{l}=\left(\frac{\partial \ln n^{-2}}{\partial z}\right)_{l}=\ln \left(n_{l-1} / n_{l+1}\right) / \Delta z
$$

This type of formula has often been used for evaluating the transverse field components for the TM wave analysis. Alternatively, we can use the following formula:

$$
n^{2} \frac{\partial}{\partial z}\left(\frac{1}{n^{2}}\right)_{l}=\frac{u_{l+1}-u_{l-1}}{\Delta z}
$$

where

$$
u_{p}=\frac{2 n_{l}^{2}}{n_{l}^{2}+n_{p}^{2}} \quad p=l+1 \quad \text { or } \quad l-1 .
$$

The latter equation has first been employed in a full-vectorial FD scheme based on the $H$-field to evaluate polarization coupling terms [18].

To compare (10) and (11), we calculate the normalized power of the asymmetrical waveguide at a propagation distance of 50 $\mu \mathrm{m}$. Note that the waveguide geometry to be considered here corresponds to the worst case (a strongly guiding structure with the largest asymmetry) studied by Vassallo [1] in terms of nonconservation of power. The data as a function of tilt angle are presented in Fig. 7, in which $\Delta x$ is chosen to be $d /(21 \cos \Theta)$, and the condition of $\Delta z=\Delta x / \tan \Theta$ is applied. The reference index is taken to be $n_{0}=\beta \cos \Theta / k_{0}$. In this model, (11) exhibits better power conservation behavior than (10). Further comparison between (10) and (11) shows that they do not yield significant difference for a waveguide with slight asymmetry. We adopt (11) in the following analysis, but (10) yields nearly the same results in the models in Figs. 9 and 11.

Fig. 8 shows a typical example of the normalized power evolution for an asymmetrical waveguide tilted by an angle of $\Theta=$ $5^{\circ}$. It is obvious that the negligence of $\partial_{z} n^{-2}$ results in power amplification, while its inclusion serves to maintain the initial power.

As an application of the present method, we discuss two specific models. We first deal with an asymmetrically tapered waveguide shown in Fig. 9. This taper was used in a benchmark test [6]. The configuration parameters are $n_{\text {core }}=3.30, n_{\text {sub }}=$ $3.17, n_{\text {cover }}=3.17$, and $\Theta=1.0^{\circ}$. A wavelength of $\lambda=$ $1.55 \mu \mathrm{m}$ is used, and $n_{0}$ is fixed to be $n_{\mathrm{sub}}$. To verify the reciprocity of the propagation, we calculate the guided-mode power as a function of propagation distance. The results are presented in Fig. 10. The end points for forward and backward propagations should be the same due to the reciprocity. It should

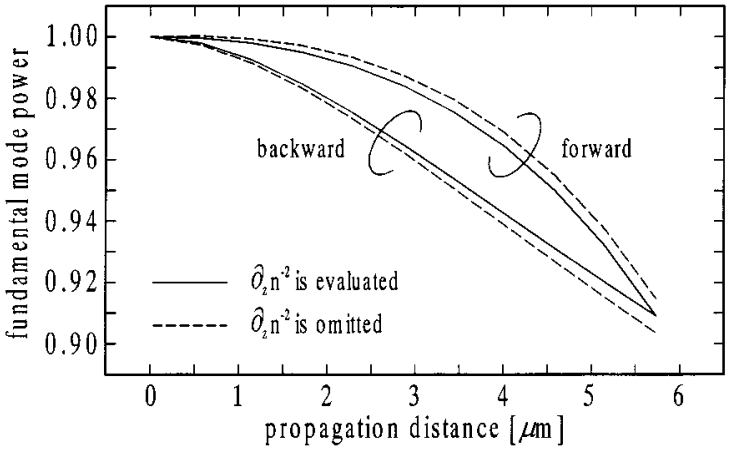

Fig. 10. Guided-mode power as a function of propagation distance.

TABLE I

SumMary OF THE Fundamental MODE POWER LOSS VALUES

\begin{tabular}{l|c|c|c|c}
\hline \multirow{2}{*}{} & \multicolumn{2}{|c|}{$\Theta=0.1^{\circ}$} & \multicolumn{2}{c}{$\Theta=1.0^{\circ}$} \\
\cline { 2 - 5 } Twente & Forward & Backward & Forward & Backward \\
\hline Thomson & 3.39 & 3.40 & 9.15 & 9.14 \\
\hline AAR-UCL & 3.17 & 3.17 & 9.43 & 9.64 \\
\hline Hagen & 3.39 & 3.45 & 9.15 & 9.20 \\
\hline IREE & 3.37 & 3.37 & 9.10 & 9.10 \\
\hline Gent & 3.37 & 3.37 & 9.13 & 9.13 \\
\hline Hosei & 3.364 & 3.367 & 9.093 & 9.093 \\
\hline
\end{tabular}

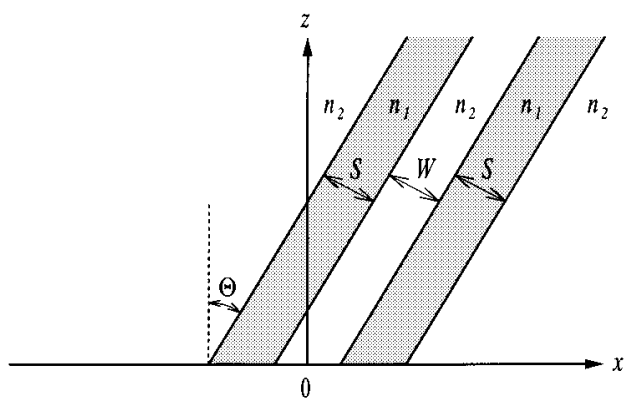

Fig. 11. Geometry of coupled waveguides placed in parallel.

be noted that discrepancy of the reciprocity is observed when $\partial_{z} n^{-2}$ is neglected. In contrast, the reciprocity is recovered when including this term. More specifically, the guided-mode power loss is calculated to be $9.093 \%$ for forward propagation, and $9.093 \%$ for backward propagation with $\Delta x=0.01 \mu \mathrm{m}$, $\Delta z=0.573 \mu \mathrm{m}$, and $N_{x}=2048$. We also calculate the case for $\Theta=0.1^{\circ}$ under the condition of the same sampling parameters. The guided-mode power loss is found to be 3.364 and $3.367 \%$ for forward and backward propagations, respectively. Detailed comparison with the data in [6] is made in Table I.

We next investigate the propagation properties of coupled waveguides shown in Fig. 11. Two identical waveguides tilted by an angle $\Theta$ are located in parallel, and one of the waveguides is launched by the fundamental mode. The coupling of 


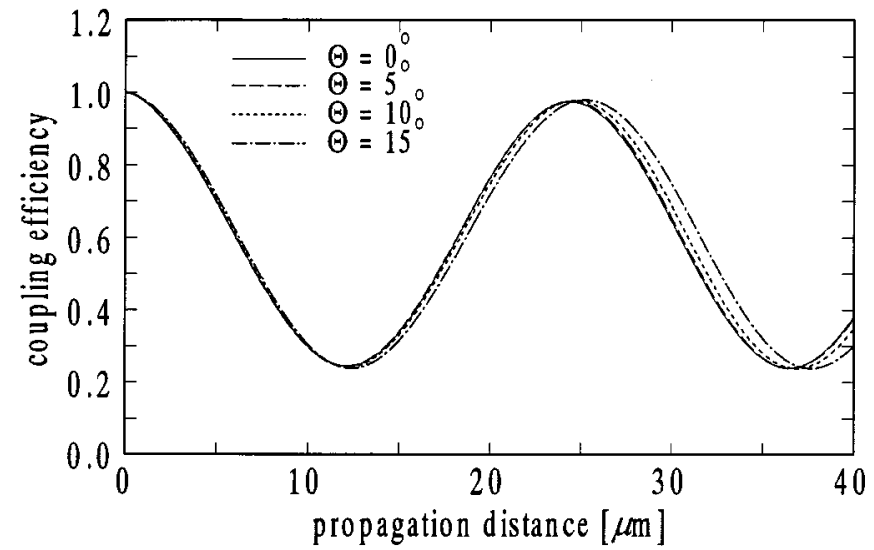

(a)

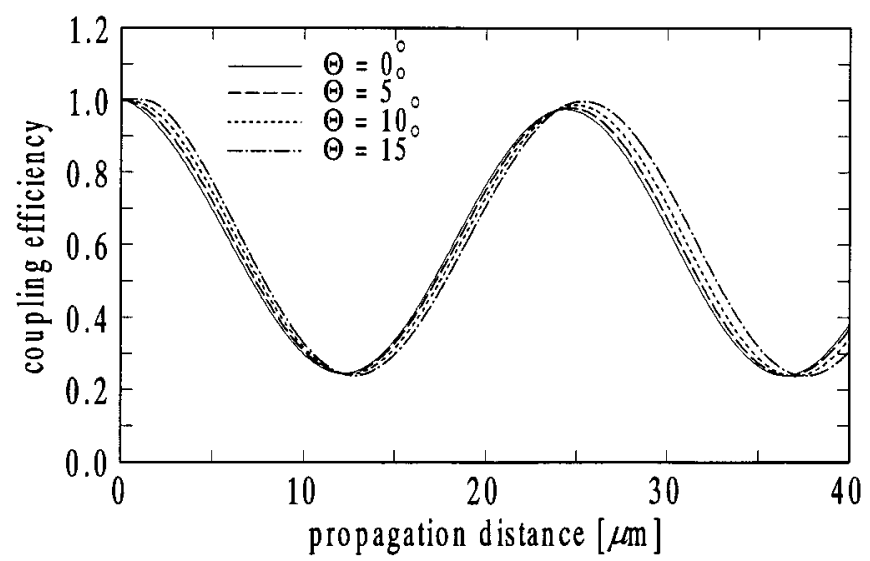

(b)

Fig. 12. Guided-mode power along the input waveguide for several tilt angles: (a) $\partial_{z} n^{-2}$ is evaluated and (b) $\partial_{z} n^{-2}$ is omitted.

the power is easily expected as the field propagates. Ideally, the coupling length should be the same regardless of the tilt angle, with the power being conserved.

To compute the coupling properties, the choice of the reference index $n_{0}$ is important. We adopt the technique of adapting $n_{0}$ after each propagation step [5], [19]. $n_{0}$ is determined by

$$
n_{0}^{2}=\frac{\int\left(k_{0}^{2}|H|^{2}-\frac{1}{n^{2}}\left|\frac{\partial H}{\partial x}\right|^{2}\right) d x}{k_{0}^{2} \int \frac{|H|^{2}}{n^{2}} d x} .
$$

The three-point finite-difference formula of (9) that takes into account the discontinuity of the first derivative is employed to evaluate $\partial H / \partial x$.

The configuration parameters of the coupled waveguides are taken to be the same as those investigated by the finite-element BPM [19], i.e., $n_{1}=1.55, n_{2}=1.3, S=0.5 \mu \mathrm{m}$, and $W=$ $0.5 \mu \mathrm{m}$. The computation parameters are $\Delta x=d /(20 \cos \Theta)$, $\Delta z=\Delta x / \tan \Theta$ except for $\Theta=0^{\circ}(\Delta z=0.1 \mu \mathrm{m}$ for $\Theta=0^{\circ}$ ), and $N_{x}=2000$. Fig. 12 shows the guided-mode power along the input waveguide for several tilt angles. The coupling length and the power remain relatively unchanged when $\Theta$ is changed. For comparison, the data without $\partial_{z} n^{-2}$ are also presented in Fig. 12(b). We can again find that power is well conserved when $\partial_{z} n^{-2}$ is included. This is particularly obvious when the guided-mode power becomes maximal.

\section{CONCLUSION}

A power (norm)-conserving beam propagation technique has been investigated for TM waves in step-index slab waveguides. We first review the necessity of including the $z$-derivative of the refractive index $\partial_{z} n^{-2}$. The effectiveness of introducing a modified finite-difference formula for a general position of an interface is demonstrated through the propagating beam analysis of a tilted symmetrical waveguide. For the analysis of an asymmetrical waveguide, $\partial_{z} n^{-2}$ must be taken into account. With this in mind, comparison between the finite-difference equations for $\partial_{z} n^{-2}$ is made. As an application, an asymmetrical taper and tilted waveguides placed in parallel are analyzed to validate the present technique.

\section{REFERENCES}

[1] C. Vassallo, "Difficulty with vectorial BPM," Electron. Lett., vol. 33, pp. 61-62, 1997.

[2] H. J. W. M. Hoekstra, "On beam propagation methods for modeling in integrated optics," Opt. Quantum Electron., vol. 29, pp. 157-171, 1997.

[3] L. Poladian and F. Ladouceur, "Unification of TE and TM beam propagation algorithms," IEEE Photon. Technol. Lett., vol. 10, pp. 105-107, 1998.

[4] C. Vassallo, "Interest of improved three-point formulas for finite-difference modeling of optical devices," J. Opt. Soc. Amer. A, vol. 14, pp. 3273-3284, 1997.

[5] F. Schmidt, "An adaptive approach to the numerical solution of Fresnel's wave equation," J. Lightwave Technol., vol. 11, pp. 1425-1434, 1993.

[6] J. Haes, R. Baets, C. M. Weinert, M. Gravert, H. P. Nolting, M. A. Andrade, A. Leite, H. K. Bissessur, J. B. Davies, R. D. Ettinger, J. Ctyroky, E. Ducloux, F. Ratovelnomanana, N. Vodjdani, S. Helfert, R. Pregla, F. H. G. M. Wijnands, H. J. W. M. Hoekstra, and G. J. M. Krijnen, "A comparison between different propagative schemes for the simulation of tapered step index slab waveguides," J. Lightwave Technol., vol. 14, pp. $1557-1569,1996$.

[7] K. S. Yee, "Numerical solution of initial boundary value problems involving Maxwell's equations in isotropic media," IEEE Trans. Antennas Propagat., vol. AP-14, pp. 302-307, 1966.

[8] T. Barts, J. Browman, R. K. Cooper, M. Dehler, M. Dohlus, F. Ebeling, A. Fischerauer, G. Fischerauer, P. Hahne, R. Klatt, F. Krawczyk, M. Marx, T. Pröndpper, G. Rodenz, D. Rusthoi, P. Schündtt, B. Steffer, T. Weiland, and S.G. Wipf, "Maxwell's grid equations," Frequenz, vol. 44, pp. 9-19, 1990.

[9] T. Itoh, G. Pelosi, and P. P. Silvestor, Eds., Finite Element Software for Microwave Engineering. New York: Wiley, 1996.

[10] J. Yamauchi, G. Takahashi, and H. Nakano, "Modified finite-difference formula for semivectorial H-field solutions of optical waveguides," IEEE Photon. Technol. Lett., vol. 10, pp. 1127-1129, 1998.

[11] — , "Full-vectorial beam-propagation method based on the McKee-Mitchell scheme with improved finite-difference formulas," $J$. Lightwave Technol., vol. 16, no. 12, pp. 2458-2464, 1998.

[12] J. Yamauchi, M. Sekiguchi, O. Uchiyama, J. Shibayama, and H. Nakano, "Modified finite-difference formula for the analysis of semivectorial modes in step-index optical waveguides," IEEE Photon. Technol. Lett., vol. 9, pp. 961-963, 1997.

[13] M. S. Stern, "Semivectorial polarized H field solutions for dielectric waveguides with arbitrary index profiles," Proc. Inst. Elect. Eng., pt. J, vol. 135, no. 5, pp. 333-338, 1988.

[14] D. Yevick and W. Bardyszewski, "Correspondence of variational finitedifference (relaxation) and imaginary-distance propagation methods for modal analysis," Opt. Lett., vol. 17, pp. 329-330, 1992.

[15] C. L. Xu, W. P. Huang, and S. K. Chaudhuri, "Efficient and accurate vector mode calculations by beam propagation method," J. Lightwave Technol., vol. 11, pp. 1209-1215, 1993.

[16] S. Jüngling and J. C. Chen, "A study and optimization of eigenmode calculations using imaginary-distance beam-propagation method," IEEE J. Quantum Electron., vol. 30, pp. 2098-2105, 1994. 
[17] G. R. Hadley, "Transparent boundary condition for beam propagation," Opt. Lett., vol. 16, pp. 624-626, 1991.

[18] C. Vassallo, "Finite difference analysis of vectorial transversal fields in optical waveguides," in Proc. 3rd Int. Conf. Mathematical and Numerical Aspects of Wave Propagation (SIAM-INRIA), Mandeilieu, 1995, pp. 594-603.

[19] Y. Tsuji, M. Koshiba, and T. Tanabe, “ A wide-angle beam propagation method using a finite element scheme," IEICE Trans., vol. J79-C-I, pp. 381-388, 1996.

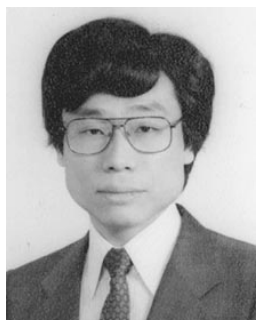

Junji Yamauchi (M'85) was born in Nagoya, Japan, on August 23, 1953. He received the B.E., M.E., and Dr.E. degrees from Hosei University, Tokyo, Japan, in 1976,1978 , and 1982, respectively.

From 1984 to 1988, he served as a Lecturer in the Electrical Engineering Department of Tokyo Metropolitan Technical College. Since 1988, he has been a Member of the Faculty of Hosei University, where he is now a Professor of Electronic Informatics. His research interests include optical waveguides and circularly polarized antennas.

Dr. Yamauchi is a member of the Optical Society of America (OSA) and the Institute of Electronics, Information, and Communication Engineers (IEICE) of Japan.

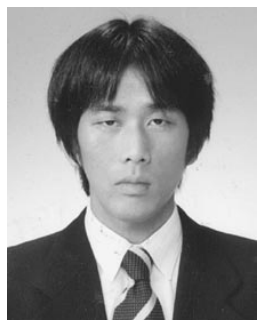

Kenji Matsubara was born in Tokyo, Japan, on September 4, 1974. He received the B.E. and M.E. degrees from Hosei University, Tokyo, Japan, in 1997 and 1999, respectively. He joined Fujitsu Co. Ltd. in 1999.

Mr. Matsubara is a member of the Institute of Electronics, Information, and Communication Engineers (IEICE) of Japan.

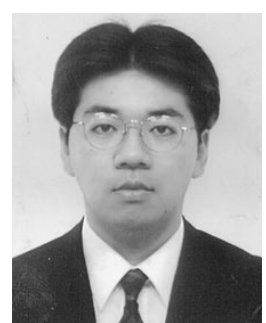

Takeshi Tsuda was born in Kanagawa, Japan, on May 14, 1974. He received the B.E. degree in 1999 from Hosei University, where he is presently in the Master's program.

Mr. Tsuda is a member of the Institute of Electronics, Information and Communication Engineers (IEICE) of Japan.

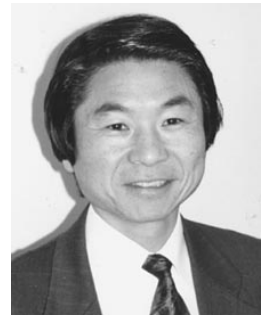

Hisamatsu Nakano (M'75-SM'87-F'92) was born in Ibaraki, Japan, on April 13, 1945. He received the B.E., M.E., and Dr.E. degrees in electrical engineering from Hosei University, Tokyo, in 1968, 1970 , and 1974, respectively.

Since 1973, he has been a Member of the Faculty of Hosei University, where he is now a Professor of Electronic Informatics. His research topics include numerical methods for antennas, electromagnetic wave scattering problems, and lightwave problems. He has published more than 130 refereed journal papers and 90 international symposium papers on antenna and relevant problems. $\mathrm{He}$ is the author of Helical and Spiral Antennas (New York: Research Studies Press, Wiley, 1987). He published the chapter "Antenna analysis using integral equations," in Analysis Methods of Electromagnetic Wave Problems, vol. 2 (Norwood, MA: Artech House, 1996). He was a Visiting Associate Professor at Syracuse University, Syracuse, NY, during May-September 1981, a Visiting Professor at the University of Manitoba, Canada, during March-September 1986, and a Visiting Professor at the University of California, Los Angeles, during September 1986-March 1987.

Dr. Nakano received an International Scientific Exchange Award from the Natural Sciences and Engineering Research Council of Canada. In 1987, he received the Best Paper Award from the IEE 5th International Conference on Antennas and Propagation. In 1994, he received the IEEE Antennas and Propagation Society's Best Application Paper Award (H. A. Wheeler Award). He is an Associate Editor of IEEE ANTENNAS AND PROPAGATION MAGAZINE. 